ISSN 0001-6002/2001/43/2/55-63

Acta Médica Costarricense, (C2001

Colegio de Médicos y Cirujanos

\title{
Lesiones Vasculares Abdominales: El Desafío del Cirujano de Trauma
}

\author{
Juan A Asensio, ${ }^{1}$ Salvador Navarro-Soto, ${ }^{1}$ Walter Forno, ${ }^{1}$ Gustavo Roldan, ${ }^{1}$ Luz María Rivas, ${ }^{1}$ Ali Salim, ${ }^{1}$ \\ Vincent Rowe, ${ }^{1}$ Demetrios Demetriades ${ }^{1}$
}

Introducción: Las lesiones vasculares abdominales presentan los mayores índices de mortalidad y morbilidad de entre todas las lesiones que puede sufrir un enfermo traumático.

Método: Revisión de la clínica, diagnóstico, vías de abordaje y tratamiento de los pacientes con lesiones vasculares intraabdominales, basada en la experiencia en el manejo de 302 enfermos.

\begin{abstract}
Resultados: Las heridas penetrantes abdominales constituyen entre el 90\%-95\% de las lesiones que afectan a los vasos abdominales. La lesión abdominal multiorgánica es frecuente. Los hallazgos clínicos compatibles con hemoperitoneo o peritonitis y la ausencia de pulsos femorales son tributarios de laparotomía. En los pacientes que presentan paro cardio-respiratorio, se debe realizar toracotomía de urgencia para masaje cardiaco abierto y pinzamiento aórtico. La mortalidad global es del 54\%, la exsanguinación representa el 85\% de la misma. El síndrome compartimental en el abdomen y en las extremidades, así como el círculo vicioso de la acidosis, la hipotermia y la coagulopatía son las principales complicaciones.
\end{abstract}

Conclusiones: Las lesiones vasculares abdominales presentan una alta mortalidad y morbilidad. El conocimiento anatómico del retroperitoneo y de las vías de abordaje de los vasos así como una exploración clínica adecuada ayudaran a disminuir las complicaciones y la mortalidad de estos pacientes.

Descriptores: lesiones vasculares abdominales, clínica, tratamiento.

Recibido: 20 de agosto de 2000.

Aceptado: 17 de abril de 2001.

Las lesiones vasculares intraabdominales, se encuentran entre las más letales que puede sufrir un paciente traumatizado, además de ser de las más difíciles de tratar y representar siempre un desafío para el cirujano moderno de trauma. La mayor parte de estos pacientes llegan a los servicios de urgencias en shock, secundario a una pérdida masiva de sangre, el cual a menudo, es irreversible. Los pacientes que han sufrido una lesión vascular abdominal, son un ejemplo claro del círcu-

Abreviaturas: FMO, fallo multiorgánico; TAC, tomografía axial computarizada; ATLS, Advanced Trauma Life Support; AAST, Asociación Americana para la Cirugía del Trauma; PTFE, politetrafluoro etileno.

1 Los Angeles County University of Southern California Medical Center.

Correspondencia: Juan A. Asencio. División de Trauma y Cuidados Intensivos, Los Angeles County University of Southern California Medical Center. 1200 North State Street, Room 10-750, Los Angeles, California 90033-4525. EE.UU. lo vicioso creado por el shock, la acidosis, la hipotermia, la coagulopatía y las arritmias cardíacas. ${ }^{1}$

Muchos de estos pacientes llegan al hospital en paro cardiorespiratorio y precisan de medidas de resucitación drásticas para intentar que lleguen con vida al quirófano, entre ellas: toracotomía en urgencias, pinzamiento aórtico y resucitación cardiopulmonar abierta. ${ }^{2-4}$ La exposición de los vasos retroperitoneales, requiere una amplia disección y mobilización de las estructuras intraabdominales, agravando aún más el problema del manejo de estos pacientes. Estas maniobras requieren tiempo y pueden ser potencialmente peligrosas, puesto que la disección rápida a través de grandes hematomas retroperitoneales es difícil y se corre el peligro de añadir patología a un paciente que no puede permitirse otras lesiones

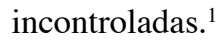

En general, las lesiones vasculares abdominales raramente se presentan aisladas, hallando con frecuencia lesiones asociadas, que aumentan la gravedad de la agresión y el tiempo 
necesario para repararlas. Estas lesiones se caracterizan por una gran pérdida sanguínea, requiriéndose grandes cantidades de cristaloides, sangre y derivados para la sustitución de la volemia. A esto se le añade la necesidad frecuente del pinzamiento aórtico o de otros grandes vasos abdominales, predisponiendo a estos pacientes al desarrollo de lesiones por isquemia-reperfusión. ${ }^{1-4}$

El concepto de cirugía de "bail-out" popularizado por Stone ${ }^{5}$ a principios de los años 80 y conocida posteriormente como cirugía de control de daños, se utiliza frecuentemente en el tratamiento de estos pacientes, ya que requieren con frecuencia cierres temporales de la pared abdominal, y necesitan reintervenciones que pueden añadir otras lesiones a un paciente ya comprometido.

Uno de los mayores dilemas al que se enfrenta el cirujano actual de trauma es cómo reparar lesiones vasculares en un medio contaminado masivamente, evitando la infección de éstas. ${ }^{7,8}$ Complicaciones tales como: shock profundo, hipoperfusión tisular, remplazo de la volemia, contaminación prolongada, procesos sépticos y fallo multiorgánico (FMO) a menudo aparecen en estos pacientes dificultando su tratamiento y conllevando a altos índices de morbilidad y mortalidad. Es evidente que los mejores resultados son obtenidos cuando el cirujano cuenta con la experiencia y el "armamentarium" quirúrgico necesario para enfrentarse a este tipo de lesiones. ${ }^{5-14}$

\section{Perspectiva Histórica}

Algunas de las primeras contribuciones al desarrollo de la cirugía vascular fueron realizadas por Eck $^{7,8,15}$ cirujano ruso, quien en 1877 realizó una anastomosis entre la vena porta y la vena cava inferior. Silberberg ${ }^{7,8,15}$ en 1897 realizó con éxito suturas arteriales incluyendo la aorta abdominal. En 1899, Dorfler ${ }^{7,8,15}$ recomendó el uso de agujas de punta redonda fina $\mathrm{y}$ de suturas que incluyeran todas las capas del vaso, teniendo éxito en 12 de 16 experimentos. De igual forma $\mathrm{Payr}^{7,8,15} \mathrm{en}$ 1900 realizó una anastomosis arterial invaginante utilizando anillos de magnesio. Otros avances fueron realizados por Clermont ${ }^{7,8,15}$ quien en 1901 practicó una anastomosis término-terminal en la cava inferior con una sutura continua de seda fina.

Outbot $^{7,8,15}$ en 1950, realizó un injerto homólogo arterial en una bifurcación aórtica trombosada. Dubost ${ }^{7,8,15}$ en 1951 resecó un aneurisma de aorta abdominal restaurando la continuidad arterial por medio de un injerto homólogo de aorta torácica. Julian, DeBakey y Szilagyi le siguieron rápidamente. ${ }^{7,8,15}$ Voorhees ${ }^{15}$ en 1956 fue el pionero de los injertos protésicos de la aorta abdominal.

\section{Incidencia}

Las lesiones vasculares abdominales ocurren con poca frecuencia en los conflictos bélicos, pero ocurren a menudo en la vida civil. En 1946, DeBakey y Simeone ${ }^{16}$ publicaron una serie de 2417 lesiones arteriales atendidas durante la
Segunda Guerra Mundial, incluyendo 49 lesiones intra-abdominales que representan un 2\%. Hughes ${ }^{17}$ en 1958 publicó 304 lesiones arteriales durante la Guerra de Corea, de las cuales solo 7 afectaron a las arterias ilíacas, con una incidencia del 2.3\%. Rich ${ }^{18}$ en 1970 publicó una serie de 1000 heridas arteriales, sufridas durante la Guerra de Vietnam, de las que 29 (2.9\%), afectaban vasos intra-abdominales.

En las series civiles, de todas las lesiones vasculares del $27 \%$ al $33 \%$ corresponden a lesiones vasculares abdominales, ${ }^{7,8}$ aumentando de forma progresiva su incidencia durante los últimos años. Asensio y colaboradores ${ }^{19}$ han publicado recientemente 302 lesiones vasculares abdominales, tratadas en el Centro de Trauma de Los Angeles County-University of Southern California en un periodo de seis años; en este centro se atienden de 7000 a 7500 víctimas de trauma al año. Demetriades, Asensio y colaboradore ${ }^{20}$ publicaron 67 casos de pacientes con lesiones penetrantes de la aorta abdominal atendidos en el mismo centro en un periodo de cinco años.

\section{Mecanismos de Lesión}

Las heridas penetrantes abdominales son la causa más común de lesiones vasculares a este nivel, representando entre el $90 \%$ y el $95 \%$ de todas las causas de lesiones vasculares intra-abdominales. El traumatismo abdominal cerrado es responsable aproximadamente sólo del 5\% al $10 \%$ restante. ${ }^{7,820-22}$ De todos los pacientes sometidos a laparotomía por heridas abdominales por arma de fuego, aproximadamente el $25 \%$ presentan lesiones vasculares, mientras que sólo el $10 \%$ de los pacientes intervenidos por heridas abdominales por arma blanca, presentan lesión vascular. ${ }^{7,820-22}$ Las lesiones abdominales penetrantes son normalmente debidas a heridas por arma blanca o arma de fuego; las contusiones abdominales son resultado de accidentes de tránsito con traumatismo directo sobre el abdomen.

\section{Lesiones Asociadas}

Debido a su localización retroperitoneal y a la proximidad de otros órganos, los vasos abdominales raramente son lesionados aisladamente, por lo que la lesión multiorgánica es más la regla que la excepción. Se ha estimado que cuando existe lesión vascular intraabdominal, ésta se asocia a una media de 2 a 4 lesiones intrabdominales. ${ }^{7,8,19,20-22}$ El traumatismo penetrante es la principal causa de lesión intra-abdominal multiorgánica asociada a lesiones vasculares intraabdominales.

Frecuentemente, varios vasos están afectados en aquellos pacientes que presentan lesión vascular intraabdominal. Una combinación de lesión arterial y venosa es la más común de las lesiones vasculares intraabdominales. ${ }^{19}$

\section{Localización Anatómica de la Lesión}

En el traumatismo abdominal cerrado, la lesión de los vasos de la parte superior es lo más frecuente. Sin embargo, las lesiones penetrantes son impredecibles y pueden ocurrir en 
cualquier parte del abdomen, pudiendo afectar como ya se ha señalado a más de un vaso. Debido a la proximidad entre las arterias y las venas abdominales, se pueden producir fístulas arterio-venosas, sin embargo su frecuencia es baja. ${ }^{7,8}$

La aorta abdominal y la vena cava inferior pueden lesionarse supra e infrarenal, sumándose en el caso de la cava, la lesión retrohepática, la cual se asocia con una alta mortalidad. La arteria mesentérica superior puede lesionarse en cualquiera de sus cuatro porciones. La vena mesentérica superior puede afectarse a nivel infrapancreático o retropancreático. La vena porta puede lesionarse en su origen, en su confluencia con la vena mesentérica superior y la esplénica, o aisladamente en el hilio hepático. La arteria renal puede lesionarse en cualquiera de sus tres porciones y la vena renal puede hacerlo a nivel de su confluencia con la vena cava o en el hilio renal. ${ }^{1}$

\section{Diagnóstico}

a. Clínica: El cirujano de trauma debe ser consciente de que cualquier lesión penetrante en el torso, desde la línea mamaria hasta los muslos, presenta un elevado riesgo de lesión vascular.

Las lesiones penetrantes en la línea media se asocian frecuentemente con lesión aórtica o de la cava. Las heridas periumbilicales pueden afectar así mismo a la aorta y la bifurcación de la cava. Las heridas por arma de fuego que atraviesan la cavidad abdominal o la pelvis también poseen un alto riesgo de lesión de estructuras vasculares. Este tipo de lesión es altamente letal. En el hipocondrio derecho la sospecha de lesión se debe dirigir hacia la aorta, la cava y el eje portal. ${ }^{1}$

La presentación clínica variará según si existe un hematoma retroperitoneal contenido o un sangrado libre en la cavidad abdominal. Obviamente en el primer caso el paciente se presentara estable hemodinámicamente o con algún grado de hipotensión que responde a la administración de líquidos, mientras que aquellos con sangrado libre peritoneal presentaran hipotensión severa. ${ }^{1,7}$

La presencia de una herida penetrante abdominal asociada a distensión y shock, indica la presencia de hemorragia libre, secundaria a lesión de un vaso grande. En pacientes con lesión pélvica penetrante y ausencia de pulsos femorales, debe pensarse en lesión arterial ilíaca ipsilateral. ${ }^{1}$

En aquellos pacientes con traumatismo abdominal cerrado, con o sin hipotensión y con hematuria macro o microscópica, el cirujano de trauma debe de sospechar lesión vesical o de vasos renales. Cualquier paciente que tenga una herida penetrante abdominal e historia de hipotensión previa a su traslado, presenta lesión vascular hasta que se demuestre lo contrario. ${ }^{1,7,8}$

El dolor abdominal y la irritación peritoneal o signos de peritonitis pueden ser debidos a lesión vascular o a lesiones frecuentemente asociadas con las mismas. ${ }^{1-4,7,8}$
La presencia o ausencia de pulso femoral, poplíteo, dorsal pedio y tibial posterior debe ser examinado y documentado en ambas extremidades. De forma rutinaria debe realizarse una exploración con doppler portátil para evaluar el flujo en estos vasos, siempre que el paciente esté hemodinámicamente estable. El índice braquio-maleolar, debe ser calculado. El doppler portátil debe ser también utilizado para comprobar el flujo venoso y establecer si la elevación de las extremidades inferiores o la compresión gemelar aumentan las señales de dicho flujo. ${ }^{1-4,7,8}$

b. Exploraciones complementarias: Los datos de laboratorio proporcionan poca ayuda en el diagnóstico inicial de las lesiones vasculares abdominales. El hemograma inicial generalmente muestra una disminución en la hemoglobina y el hematocrito. La gasometría arterial determinará el pH inicial, la presencia de acidosis, la oxigenación y ventilación del paciente. El control de los gases arteriales ayudará a monitorizar el proceso de resucitación. En los pacientes hipotensos, es conveniente obtener valores basales de ácido láctico y monitorizarlos para evaluar el progreso de la resucitación. ${ }^{1}$

El ultrasonido abdominal es útil para detectar líquido libre, pero ofrece escasa información acerca del retroperitoneo y sus vasos. Una radiografía simple de abdomen puede ser de ayuda en aquellos pacientes con heridas penetrantes por arma de fuego, pues ayuda a establecer la localización y posible trayectoria de la bala. Puede realizarse un pielograma en urgencias o en el quirófano, siempre y cuando el paciente esté estable, con el fin de comprobar la función de ambos riñones. ${ }^{1}$

Una tomografia computarizada (TAC) debe realizarse en aquellos pacientes estables hemodinámicamente con o sin hematuria y que han sufrido un traumatismo abdominal, para detectar hematomas retroperitoneales o la falta de eliminación renal del medio de contraste, secundaria a lesión de los vasos renales. La angiografía sigue siendo el "Gold Standard". Sin embargo, frecuentemente no es realizable en el manejo agudo de estos pacientes. La angiografía siempre será imprescindible para el diagnóstico y tratamiento de las complicaciones postoperatorias. ${ }^{1}$

\section{Manejo Quirúrgico}

a. En urgencias: Todos los pacientes traumatizados deben ser evaluados y resucitados mediante los protocolos del ATLS (Advanced Trauma Life Support). La atención rápida dirigida a proveer una vía aérea adecuada, accesos venosos, colocación de sonda nasogástrica y sondaje vesical, así como un rápido reemplazo de volumen con Lactato de Ringer y sangre son los pilares para una correcta evaluación inicial y adecuada resucitación. En aquellos pacientes en los que existe sospecha alta de lesión vascular abdominal, no es recomendable la colocación de vías femorales, ya que puede haber lesión de las venas ilíacas o de la cava sangrando activamente, lo que evita que el volumen de reemplazo llegue al lado derecho del corazón. ${ }^{1-4,7-8}$ De igual forma, la necesidad 
de pinzar las venas ilíacas y la cava inferior durante la laparotomía, evitará que el reemplazo de volumen llegue a las cavidades cardíacas derechas, en caso de haberse canalizado las venas femorales. Así pues, catéteres gruesos deben de ser insertados en las extremidades superiores, si es preciso deben utilizarse las vías subclavia o yugular interna.

Los hallazgos clínicos compatibles con hemoperitoneo o peritonitis, y la ausencia de pulsos femorales son indicaciones para hacer una laparotomía exploradora. Deben administrarse antibióticos de amplio espectro de forma profiláctica, nuestro grupo utiliza de forma rutinaria cefoxitina. ${ }^{1-4,7-8}$

En aquellos pacientes con paro cardiorespiratorio o shock profundo, refractario a la reposición de líquidos, se debe realizar una toracotomía de urgencia para dar masaje cardiaco abierto y pinzamiento de la aorta descendente; esto último permitirá la redistribución del volumen intravascular restante mejorando la perfusión de ambas carótidas y de las arterias coronarias, disminuyendo o deteniendo a su vez la hemorragia arterial intraabdominal. ${ }^{1-4,7,8,23,24}$

La toracotomía de urgencia y el pinzamiento de la aorta descendente, somete al paciente a riesgos como: isquemia distal, hipotermia secundaria al "torax abierto" y predisposición a las lesiones por reperfusión. A pesar de estos riesgos, es una maniobra útil y con frecuencia la última esperanza, para aquellos pacientes en los cuales el control de la hemorragia no pueda obtener de forma inmediata. Sobra decir, que en estos pacientes el tiempo es esencial y deben ser rápidamente llevados al quirófano sin exploraciones complementarias o retrasos. ${ }^{1-4,7,8,23,24}$

b. Manejo Intraoperatorio: En el quirófano el paciente debe ser preparado desde el cuello hasta los muslos. La cara interna de esta zona es importante, dada la posibilidad de que sea necesario obtener un injerto de safena. El cirujano debe confirmar que haya sangre preparada para una transfusión rápida. También debe evitarse en lo posible la hipotermia del paciente, mediante la aplicación de mantas en la mesa de operaciones, cubriendo las extremidades inferiores y la cabeza, con colchones de aire caliente, aumentando la temperatura del ventilador a $42 \mathrm{C}$ y teniendo a disposición líquidos calientes. La posibilidad de contar con un aparato de autotransfusión puede ser de gran ayuda. ${ }^{1-4,7,8}$

Las lesiones abdominales deben ser exploradas a través de una laparotomía media xifopúbica. El control inmediato de la hemorragia exsanguinante y de la fuente de contaminación intraabdominal, en caso de que exista, son los objetivos inmediatos que deben conseguirse, seguido por una minuciosa exploración del resto de la cavidad abdominal. El retroperitoneo debe ser explorado de forma sistemática, puesto que las estructuras vasculares se localizan en esta zona, lo que exige un profundo conocimiento anatómico de la región por parte del cirujano..$^{1,7,8}$

Como ya hemos mencionado, el principal objetivo en el manejo de las lesiones vasculares abdominales es el control de la hemorragia, mediante la disección proximal y distal del vaso lesionado. Sin embargo, en las hemorragias vasculares exsanguinantes conseguirlo rápidamente puede ser difícil. ${ }^{1-4}$

Con frecuencia estos pacientes presentan severa hipotensión, así pues, el pinzamiento de la aorta es la primera maniobra capaz de detener la hemorragia que amenaza su vida. Si éste llega hipotenso y sufre paro cardiorespiratorio en el quirófano, debe procederse a una toracotomía anterolateral izquierda y pinzamiento aórtico, prosiguiendo después con la laparotomía. ${ }^{1-4,23,24}$

En los casos en los cuales el paciente llega estable hemodinámicamente, pero se descompensa durante la laparatomía, la aorta abdominal puede ser controlada digitalmente a nivel del hiato o bien se puede utilizar un compresor de aorta o realizar un pinzamiento. La colocación de la pinza oclusiva vascular en esta área puede ser difícil por la presencia de los pilares diafragmáticos, requiriendo a veces la sección de los mismos. ${ }^{1-4,23,24}$

Una vez que la hemorragia ha sido controlada el cirujano debe ubicarla, en una de las tres zonas del retroperitoneo, de igual forma debe actuarse en el caso de los hematomas retroperitoneales. Existen tres zonas en el espacio retroperitoneal, Zona I, II y III. Para el cirujano es imprescindible conocer la intrincada anatomía de estas zonas. La Zona I empieza en el hiato aórtico y acaba en el promontorio sacro, se encuentra en la línea media sobre los cuerpos vertebrales. Esta zona se divide en zona I supramesocólica y zona I inframesocólica. Hay dos zonas II, derecha e izquierda, localizadas en los espacios paracólicos. La zona III empieza en el promontorio sacro y va hacia la pelvis. ${ }^{1-4,7-8}$

La zona I supramesocólica, contiene la aorta abdominal suprarrenal, el tronco celíaco y las dos primeras partes de la mesentérica superior, esta se divide en sub-zona 1 desde su origen en la aorta hasta el origen de la pancreaticoduodenal inferior, sub-zona 2 desde ésta hasta el origen de la cólica media, la sub-zona 3 es el tronco distal a la arteria cólica media y la sub-zona 4 está constituidas por las ramas segmentarias yeyunales, ileales y cólicas. Esta zona I supramesocólica también contiene la vena cava inferior infrahepática suprarrenal y la parte proximal de la vena mesentérica superior. La zona I inframesocólica contiene: la aorta abdominal infrarenal, la vena cava infrarenal, la arteria mesentérica inferior, las zonas 3 y 4 de la arteria mesentérica superior y la parte distal de la vena mesentérica superior.

Las zonas II derecha e izquierda, contienen los pedículos vasculares renales. La zona III, contiene las arterias y venas ilíacas primitivas, así como sus ramas externas e internas y el plexo pressacro. La zona portal, contiene la vena porta, la arteria hepática y la vena cava inferior retrohepática. ${ }^{1-4,7,8}$

Tan pronto como el cirujano ha identificado y localizado la hemorragia o el hematoma retroperitoneal en alguna de las zonas mencionadas, debe abordar la zona intentando obtener el control del vaso lesionado para exponerlo y realizar la 
reparación. Cada zona requerirá de maniobras de diferente grado de complejidad para la exposición de los vasos.

La zona I supramesocólica, es generalmente abordada realizando una maniobra que rota medialmente las vísceras localizadas en el lado izquierdo. Este abordaje requiere la disección de la línea avascular de Toldt en el colon izquierdo, junto con la incisión del ligamento esplenorrenal, de esta forma el colon descendente, el bazo, el cuerpo y cola del páncreas y el estómago pueden ser rotados medialmente. Con esta maniobra se consigue exponer: la aorta desde su entrada a la cavidad abdominal por el hiato, el origen del tronco celíaco, la arteria mesentérica superior y el pedículo renal izquierdo. Es posible movilizar el riñón izquierdo medialmente, pero es una maniobra que generalmente no se hace. ${ }^{1-4,7-8}$

De forma alternativa se puede realizar una maniobra de Kocher y la disección de la línea avascular de Toldt en el colon ascendente, lo que permitirá movilizar medialmente el colon derecho, la flexura hepática, duodeno y cabeza del páncreas a nivel de los vasos mesentéricos superiores; además se debe incidir el tejido retroperitoneal a la izquierda de la vena cava inferior. Esta maniobra expone la aorta abdominal suprarenal, entre el tronco celíaco y la arteria mesentérica superior; pero tiene la desventaja de que se obtiene una exposición por debajo del nivel de algunas lesiones de la aorta supracelíaca en el hiato. ${ }^{1-4,7-8}$

Las maniobras utilizadas para exponer la Zona I inframesocólica, deben desplazar el colon transverso cranealmente, eviscerando el intestino delgado hacia la derecha, seccionando el ligamento de Treitz y el tejido areolar a la izquierda de la aorta abdominal hasta localizar la vena renal izquierda. Así se consigue la exposición de la aorta infrarenal. Para exponer la vena cava infrarenal, se debe seccionar la fascia de Toldt derecha y practicar una maniobra de Kocher, llevando el páncreas y el duodeno hacia la izquierda, para posteriormente, incidir el tejido retroperitoneal que cubre la vena cava inferior. ${ }^{1-4,7-8}$

La exposición de las zonas II, derecha e izquierda, dependerá de si existe un hematoma o sangrado activo en localización medial o lateral. Si se halla un hematoma en expansión o un sangrado activo medial, es preferible realizar el control del pedículo vascular renal. En el lado derecho, esto se consigue mediante la movilización del colon derecho y la realización de una maniobra de Kocher que exponga la vena cava infrarenal, para continuar la disección cranealmente incidiendo el tejido que cubre la vena cava inferior suprarenal infrahepática. Esta disección se prolongará hasta hallar la vena renal derecha, si se extiende en dirección cefálica y posteriormente localizar la arteria renal derecha. ${ }^{1-4,7,8}$

En el lado izquierdo, se movilizan el colon izquierdo y su ángulo esplénico, el intestino delgado se eviscera hacia la derecha, se localiza el ligamento de Treitz y el colon transverso y el mesocolon se desplazan cranealmente. Esto debe localizar la aorta infrarenal, una disección posterior craneal localizará la vena renal izquierda cuando cruza sobre la aorta.
La arteria renal izquierda, también se hallará en posición superior y posterior a la vena renal. En otras ocasiones, si existe un hematoma o sangrado activo a nivel lateral de la zona II, derecha o izquierda, sin extensión al hilio renal, se practicará una incisión lateral de la fascia de Gerota, desplazando medialmente el riñón pudiendo así obtener la localización del sangrado. ${ }^{1-4,7-8}$

La exposición de los vasos de la Zona III, se consigue mediante la incisión bilateral de la fascia de Toldt y desplazando medialmente tanto el colon derecho como el izquierdo; de esta forma los vasos iliacos pueden ser localizados rápidamente, junto al uréter al cruzar sobre la arteria ilíaca. Se debe colocar un tutor alrededor del uréter para retraerlo. La disección se realiza caudalmente abriendo el tejido retroperitoneal que se localiza por encima de los vasos. ${ }^{1-4,7-8}$

Una vez que se ha obtenido el control proximal y distal, todas las lesiones vasculares deben de ser clasificadas mediante la "Escala de valoración de lesiones de la Asociación Americana para la Cirugía del Trauma" para las lesiones vasculares (AAST-OIS) según se muestra en la Cuadro $1 .{ }^{25}$

Los principios básicos de la cirugía vascular deben ser sin duda aplicados para el manejo correcto de estas lesiones. Exposición adecuada, control proximal y distal, limpieza de la pared vascular dañada, prevención de la embolización por coagulos, irrigación con suero salino heparinizado, uso juicioso de los catéteres de Fogarty, suturas vasculares con monofilamento, evitar las estenosis de los vasos durante su reparación, colocación de injertos autógenos o protésicos cuando sean necesarios y realización de la arteriografía periperatoria cuando sea posible, constituyen todos los pilares de una reparación satisfactoria. ${ }^{1-4,7-8}$

El manejo de las lesiones vasculares de la Zona I, supramesocólica, consistirá en la arteriorrafia primaria de la aorta suprarrenal cuando sea posible y ocasionalmente la colocación de una prótesis de Dacrón o politetrafluoroetileno (PTFE). Las lesiones del tronco celíaco se acostumbran a tratar mediante ligadura simple.

Las lesiones de zonas 1 y 2 de la arteria mesentérica superior deben ser tratadas mediante reparación primaria, siempre que sea posible, pero frecuentemente la intensa vasoconstricción que existe lo hace difícil. Teóricamente estas lesiones pueden también ser tratadas mediante ligadura simple, pues existen suficientes colaterales capaces de preservar la irrigación del intestino delgado y del colon. Sin embargo, el profundo vasoespasmo que existe puede producir isquemia y necrosis posteriormente del intestino., ${ }^{1,19,26}$ Estas dos primeras zonas de la arteria mesentérica superior pueden ser también tratadas mediante injerto autólogo o protésico. También se ha descrito la colocación de un shunt temporal como se muestra en la Cuadro 2. ${ }^{26}$

El tratamiento de las lesiones de la zona I inframesocólica incluye las mismas técnicas que las empleadas en la zona I supramesocólica. Las zonas 3 y 4 de la arteria mesentérica 


\section{Cuadro 1 \\ "Organ Injury Scaling" de la Asociación Americana de la Cirugía del Traumatismo Escala de lesiones vasculares abdominales}

Grados Lesiones

Grado I Ramas innominadas de la arteria y vena mesentérica superior. Ramas innominadas de la arteria y vena mesentérica inferior. Arteria/vena frenica. Arteria/vena lumbar. Arteria/vena gonadal. Arteria/vena ovárica. Arteriolas o venas innominadas que requieran ligadura.

Grado II Arteria hepática, común, derecha e izquierda. Arteria/vena esplénica. Arteria gástrica derecha e izquierda. Arteria gastroduodenal. Arteria/Vena mesentérica inferior. Ramas principales de la arteria mesentérica superior y vena mesentérica inferior. Otros vasos abdominales con nombre propio que requieran ligadura/reparación.

Grado III Vena mesentérica superior. Arteria/Vena renal. Arteria/Vena llíaca. Arteria/Vena Hipogástrica. Vena cava infrarenal.

Grado IV Arteria mesentérica superior. Tronco celiaco. Vena cava suprarenal e infrahepática. Aorta infrarenal.

Grado V Vena porta. Venas hepaticas extraparenquimatosas. Vena cava, retrohepática o suprahepática. Aorta suprarenal, subdiafragmatica.

Esta clasificación se aplica a las lesiones vasculares extraparenquimatosas. Si la lesión vascular está a menos de 2 cm del parenquima, se debe acudir al "organ injury scale" específico para ese órgano. Aumentar un grado para lesiones múltiples de grado III o IV que afecten $>50 \%$ de la circunferencia del vaso. Disminuir un grado para lesiones múltiples lacrerantes de grado IV o V que afecten $<25 \%$ de la circunferencia del vaso. superior deben ser también reparadas, pero las ramas yeyunales y cólicas de la zona 4 pueden ser ligadas.,19,26 El manejo de la lesión de la arteria mesentérica inferior, consiste en su ligadura.

Las lesiones de la vena cava infrahepática suprarenal así como las de la vena cava infrarenal se tratan mediante venorrafia, siempre que sea posible. Si existe una lesión ántero posterior, se deben reparar ambas caras, poniendo a prueba la habilidad del cirujano. ${ }^{1,19}$ Aunque la vena cava inferior infrahepática suprarenal, no tiene ramas tributarias, es difícil de movilizar. En general cuando debe ser reparada una lesión ántero posterior a este nivel debe abrirse la cara anterior del vaso y desde allí, reparar la cara posterior. El vaso puede ser movilizado rotando el riñón derecho hacia afuera, sacándolo de la fosa renal, pero esta maniobra es peligrosa y no es recomendable. ${ }^{1,19}$

Cuando existe una destrucción masiva de la vena cava inferior infrahepática suprarrenal, se debe considerar su ligadura simple, aunque tras esta maniobra las tasas de supervivencia son bajas. Raramente se han utilizado prótesis en estos casos. El manejo de las lesiones de la vena cava inferior infrarrenal consiste en la venorrafia. Cuando existen lesiones ántero posteriores, la reparación primaria se puede conseguir mediante la rotación del vaso o bien mediante la técnica descrita previamente de abrir la cara anterior para reparar la posterior. La rotación del vaso es difícil, pues exige la ligadura de las venas lumbares que son sumamente frágiles, nosotros recomendamos efectuar la reparación mediante la apertura de la cara anterior. La vena cava inferior infrarrenal puede ser ligada en casos de destrucción masiva, su ligadura es por regla general bien tolerada. Las lesiones que afectan a la vena mesentérica superior deben de ser tratadas mediante reparación primaria aunque puede ser ligada pero con serias secuelas para la circulación venosa del intestino delgado y grueso. $^{1,19}$

Las lesiones de las Zonas II, derecha e izquierda, son también desafiantes. Las lesiones de la arteria renal pueden ser tratadas mediante reparación primaria o mediante colocación de injertos autólogos o protésicos. Raramente se practicará un puente aorto renal distal a la lesión.

\section{Cuadro 2 \\ Clasificación Anatómica de Fullen de las lesiones de la arteria mesentérica superior Zonas y Grados}

\begin{tabular}{|c|c|c|c|c|}
\hline Zona & Segmento A. Mesentérica Sup. & Grado & Isquemia & Segmento intestinal afecto \\
\hline 1 & $\begin{array}{l}\text { Tronco proximal a la primera rama mayor } \\
\text { (pancreatico-duodenal inferior) }\end{array}$ & 1 & Máxima & Yeyuno, ileon, colon derecho \\
\hline 2 & $\begin{array}{l}\text { Tronco entre páncreatico-duodenal } \\
\text { inferior y cólica media }\end{array}$ & II & Moderada & Gran parte intestino D, y/o colon D. \\
\hline 3 & Tronco distal a cólica media & III & Mínima & Pequeña parte ID o colon D \\
\hline 4 & Ramas yeyunales, ileales o cólicas & IV & Ninguna & No isquemia intestinal \\
\hline
\end{tabular}


Las reparaciones de las arterias renales son en general difíciles, frecuentemente se realizan ligaduras que exigen una nefrectomía. Las lesiones de las venas renales se pueden tratar mediante venorrafia primaria o ligadura simple. La ligadura de una vena renal derecha puede exigir la nefrectomía si no existen suficientes colaterales. La ligadura de la vena renal izquierda es por lo general bien tolerada, a condición de que sea realizada proximalmente y muy cerca de la cava inferior, asegurando el retorno venoso a través de las gonadales y de las renolumbares. ${ }^{1,19}$

Las lesiones de la Zona III son también difíciles de tratar puesto que con frecuencia se asocian a lesiones colónicas o genitourinarias altamente contaminantes. Las lesiones de la arteria ilíaca primitiva pueden ser reparadas mediante arteriorrafia, ocasionalmente se puede realizar resección parcial y anastomosis. También se han utilizado injertos autólogos y protésicos. Las lesiones de las arterias ilíacas internas se tratan mediante ligadura simple; las de la arteria ilíaca externa se tratan mediante arteriorrafia y ocasionalmente mediante resección y anastomosis. Se puede realizar un puente ileofemoral autólogo o protésico, aunque es infrecuente hallar una safena del adecuado calibre para hacer un puente autólogo satisfactorio. ${ }^{1,19}$

Cuando ha habido una destrucción masiva de la arteria ilíaca primitiva puede ser necesario practicar una ligadura simple, el flujo arterial puede ser reestablecido utilizando un puente femorofemoral o axilofemoral. Esta técnica tiene la desventaja de tener que utilizar vasos no lesionados y tiene una alta incidencia de trombosis. Las lesiones de las venas ilíacas primitivas, externas o internas, pueden ser tratadas satisfactoriamente mediante ligadura simple, aunque las venorrafias pueden ser también utilizadas. Ocasionalmente, el acceso a una vena ilíaca externa lesionada puede requerir la transección de la arteria ilíaca externa ipsilateral ya que la primera está por detrás de la arteria. ${ }^{1,19}$

Cada vez que un cirujano realiza una reparación de una lesión vascular abdominal, se debe tener en cuenta la posibilidad de reoperar para examinar la reparación (second look) para asegurar la viabilidad intestinal. La contaminación a través del tracto digestivo o genitourinario conlleva un gran riesgo de infección de las prótesis vasculares utilizadas para reparar los vasos lesionados, siempre que sea posible los injertos autólogos o protésicos deben ser reperitonealizados. De igual forma, es conveniente interponer tejido viable, generalmente epiplón entre todas las reparaciones vasculares realizadas cerca de una anastomosis gastrointestinal, para prevenir fístulas vásculo-entéricas y dehiscencia de sutura a posteriori. ${ }^{1}{ }^{19,26}$

\section{Mortalidad}

Las lesiones vasculares abdominales arrastran un alto índice de mortalidad; ésta puede dividirse en mortalidad precoz y tardía. La exsanguinación es la primera causa de muerte precoz en estos pacientes, es bien conocido que aquellos pacientes que llegan en shock, tienen los índices de mortalidad más altos. ${ }^{1-4}$
Asensio $^{2-4}$ reportó que la incidencia de exsanguinación en las heridas penetrantes de la aorta abdominal (suprarenal e infrarenal) es del 55\%. Además describió una incidencia de exsanguinación del $25 \%$ para las lesiones penetrantes que afectan a la arteria mesentérica superior y un $37 \%$ de incidencia de exsanguinación tanto para las lesiones penetrantes o cerradas abdominales que afectan a la arteria mesentérica superior. ${ }^{2-4}$ En una revisión de la literatura, Asensio ${ }^{2-4}$ publicó una incidencia de exsanguinación del 33\% tanto para los traumatismos abiertos como los cerrados que afectan a la vena cava inferior; así mismo comunicó una incidencia del $30 \%$ de exsanguinación, tanto para las heridas, como para las contusiones que afectan a la vena porta.

En una serie de 302 pacientes con lesiones vasculares abdominales tratados en el Los Angeles County University of Southern California (LAC + USC) Medical Center, ${ }^{19}$ durante un periodo de 72 meses, se encontraron 266 pacientes (88\%) con lesiones abdominales penetrantes: $216(81 \%)$ por arma de fuego, $46(17 \%)$ por arma blanca y $4(2 \%)$ tenían heridas por arma de fuego producidas por escopeta. Treinta y seis (12\%) ingresaron por traumatismo abdominal cerrado: 23 (64\%) sufrieron accidentes de tránsito, 11 (31\%) fueron atropellados y $2(5 \%)$ sufrieron lesiones por precipitación. Los hallazgos quirúrgicos revelaron que 275 pacientes (91\%) presentaban un hematoma retroperitoneal, 137 en la Zona I (55 supramesocólica y 82 inframesocólica), 49 en la Zona II y 89 en la Zona III. Además, 39 pacientes presentaban hematomas retroperitoneales en más de una zona. Hubo un total de 504 vasos lesionados, con un promedio de 1.67 vasos lesionados por paciente, 238 fueron lesiones arteriales (47\%) y 266 (53\%) fueron lesiones venosas. La aorta fue la arteria que se lesionó con mayor frecuencia, 60 casos $(25 \%)$. La vena lesionada con mayor frecuencia fue la cava, 77 casos (31\%), seguida por la vena mesentérica superior, 33 casos (13\%).

La mortalidad global fue del $54 \%$ y en la misma serie el $15 \%$ de los pacientes que presentaban una lesión vascular abdominal, murieron sin podérseles efectuar algún control vascular. En esta serie, se practicó toracotomía en urgencias a 43 pacientes (14\%), de los cuales sobrevivió solo uno (2\%). A 88 pacientes $(29 \%)$ se les practicó toracotomía de urgencias en el quirófano, sobreviviendo nueve (10\%), esta elevada mortalidad nos demuestra la gravedad de estos pacientes. Si excluimos los pacientes a los que se practicó toractomía urgente del análisis de mortalidad, esta disminuye hasta el $39 \%$. Los índices de mortalidad aumentaron cuando hubo más de un vaso lesionado. En estas series, la exsanguinación representa el $83 \%$ de la mortalidad global. ${ }^{1-4,} 19$

\section{Complicaciones}

Las lesiones vasculares abdominales tienen una alta morbilidad. El síndrome compartimental abdominal aparece con frecuencia en pacientes con lesiones vasculares. La incidencia de complicaciones como la trombosis, la dehiscencia de suturas y de infecciones, no es nada despreciable. La oclusión vascular es frecuente cuando la reparación se ha efectuado 
en presencia de vasoconstricción, como es habitual, al reparar las arterias renales o la arteria mesentérica superior. ${ }^{1-4,19,26}$

El síndrome hipovolémico sistémico e hipervolémico intestinal es común cuando la vena porta, la vena mesentérica superior o la cava inferior suprarenal han sido ligadas y existe poco retorno venoso desde la circulación intestinal y poco tiempo para el desarrollo de circulación venosa colateral. Pueden desarrollarse fístulas aortoentéricas, si no se interpone tejido viable entre la reparación aórtica o la intestinal. ${ }^{1,7,8}$

El círculo vicioso de hipotermia, acidosis, coagulopatía y arritmias cardíacas se presenta habitualmente en las lesiones vasculares abdominales.

La isquemia de las extremidades y los síndromes compartimentales pueden ocurrir en aquellos pacientes en los cuales se ha retrasado la restauración del flujo arterial. La misma complicación puede ocurrir en los pacientes en los que debido a una mala circulación venosa colateral, no toleran la ligadura de la vena cava inferior o las venas ilíacas primitivas. ${ }^{1,7}$

Asensio $^{19}$ en la serie de 302 pacientes con lesiones vasculares abdominales, comunica un total de 128 complicaciones. La estancia media en la UCI fue de 4 días (rango 1-67) y la estancia media hospitalaria fue de 9 dias (rango 1-45).

\section{Abstract}

Aim: Abdominal vascular injuries have a very high mortality and morbidity rates among trauma patients.

Methods: This is a clinical review of the diagnosis, surgical procedures, and treatment of patients with abdominal vascular injuries, based on the clinical experience from 302 patients presenting this type of injury.

Results: Penetrating injuries account for $90 \%$ to $95 \%$ of all abdominal vascular injuries. Mutiple organ injuries are frequent. The clinical sings of a hemoperitoneum and or peritonitis and the absence of femoral pulses are indications for laparatomy. In the patients with cardio-pulmonary arrest, an emergent thoracatomy with open cardiac mássage and cross clamping of the aorta should be performed. The global mortality rate is $54 \%$. Exsanguination accounts for $85 \%$ of these deaths. The compartment syndrome of the abdomen and limbs, as well as the cronic cycle of ascidosis, hypothermia, and coagulopathies are the principle complications.

Conclusions: Abdominal vascular injuries have a high mortality/morbidity rate. The precise knowledge of the retroperitoneal anatomy and the surgical approaches to access these vessels, as well as the adequate clinical evaluation will assist in lowering the mortality and complications rates of these patients.

Key Words: Abdominal vascular injuries, diagnosis, surgical procedures.

\section{Referencias}

1. Asensio JA, Lejarraga M. Abdominal Vascular Injury. In: Trauma Handbook. Demetriades D, Asensio JA Eds. Landes Biosciences Co. Austin, Tx. In Press

2. Asensio JA, Hanpeter D, Gomez H, Chahwan S, Orduna S, McDuffie L. Exsanguination In: Textbook of Critical Care. Shoemaker W, Greenvik A, Ayres SM, Holbrook PR, Eds. 4th Ed, Chapter 4, p37-47. W.B. Saúnders Co. Philadelphia, PA.

3. Asensio JA. Exsanguination from Penetrating Injuries. Trauma Quarterly, Urban Trauma Issue II. Buckman RF Jr, Mauro L Eds.1989; 6 (2): $1-25$.

4. Asensio JA, Lerardi R. Exsanguination. In Emergency Care Quarterly: Evolving Issues in Emergency and Trauma Care. Jacobs LM, Jr, Bennett-Jacobs B. Eds Britt LD, Guest Editor 1991; 7 (3): 59-75.

5. Stone HH, Strom PR, Mullins RJ. Management of the major coagulopathy with onset during laparotomy. Ann Surg 1983; 197: 532.

6. Rotondo MF, Schwab CW, McGonigal MD, et al. "Damage control": An approach for improved survival in exsanguinating penetrating abdominal injury. J Trauma 1993; 35:375.

7. Feliciano DV. Abdominal Vessels. In: The Textbook of Penetrating Trauma. Ivatury R, Cayten CG, Eds. Williams and Wilkins, Baltimore, MA, 1996;

8. Feliciano DV, Burch JM, Graham JM. Abdominal Vascular Injury. In Trauma. Mattox KL, Feliciano DV, Moore EE. 4th Edition McGraw Hill, 1999; Chapter 35, p783-805.

9. Feliciano DV, Burch JM: Towel clips, silos, and heroic forms of wound closure. In Maull KI, Clevland HC, Feliciano DV, et al (eds): Advances in Trauma and Critical Care. Year Book Medical Publishers, 1991; 6: 231.

10. Burch JM, Moore EE, Moore FA, et al. The abdominal compartment syndrome. Surg Clin North Am 1996; 76:88.

11. Burch JM, Ortiz V, Richardson RJ, et al. Abbreviated laparotomy and planned reoperation for critically injured patients. Am Surg 1992; 215:476.

12. Morris JA Jr, Eddy VA, Binman TA, et al. The staged celiotomy for trauma. Issues in unpacking and reconstruction. Ann Surg 1993; 217: 576.

13. Moore EE. Staged laparotomy for the hypothermia, acidosis, and coagulopathy syndrome. Am J Surg 1996; 172: 405.

14. Moore EE, Burch JM, Franciose RJ, et al. Staged physiologic restoration and damage control surgery. World J Surg 1998; 22: 1184.

15. Rich NM. Vascular Trauma. In: Surgical Clinics of North America. 1973; 53 (6): 1367-1392.

16. DeBakey ME, Simeone FA. Battle injuries of the arteries in World War II: An analysis of 2,471 cases. Ann Surg 1946; 123: 534.

17. Hughes CW. Arterial repair during the Korean War. Ann Surg 1958; 147: 555.

18. Rich NM, Baugh JH, Hughes CW. Acute arterial injuries in Vietnam: 1,000 cases. J Trauma 1970; 10: 359.

19. Asensio JA, Chahwan S, Hanpeter D, Demetriades D, Forno W, Gambaro E, et al. Operative Management and Outcome of 302 Abdominal Vascular Injuries. AAST-OIS Correlates well with mortality. Southwestern Surgical Congress Abstract. Accepted American Journal of Surgery. 
20. Demetriades D, Theodoru D, Murray J, Asensio JA, Cornwell EE, Velmahos G, et al. Mortality and Prognostic factor in Penetrating Injuries of the Aorta. J Trauma 1996; 40 (5); 761-73.

21. Feliciano DV, Bitondo CG, Mattox KL, et al. Civilian trauma in the 1980's. A 1-year experience with 456 vascular and cardiac injuries. Ann Surg 1984; 199: 717.

22. Mattox KL, Feliciano DV, Burch J, et al. Five thousand seven hundred sixty cardiovascular injuries in 4459 patients. Epidemiologic evolution 1958 to 1987. Ann Surg 1989; 209: 698.

23. Asensio JA, Voystock J, Khatri VJ, Kerstein MD. Toracotomía en el Centro de Urgencias. In Procedimientos en el Paciente Critico. 2nd Ed. Gutierrez-Lizardi, P. ED. Chapter III, p337-341. Ediciones Cuellar, Monterrey, Mexico, 1993.
24. Asensio JA, Hanpeter D, Demetriades D. The Futility of Liberal Utilization of Emergency Department Thoracotomy. Proceedings of the American Association for the Surgery of Trauma 58th Annual Meeting, p210, September 1998, Baltimore, Maryland.

25. Moore EE, Cogbill TH, Jurkovich GJ, et al. Organ Injury Scaling III: Chest wall, abdominal vascular, ureter, bladder and urethra. J Trauma 1992; 33 (3): 337-339.

26. Asensio JA, Berne JD, Chahwan S, Hanpeter D, Demetriades D, Velmahos GC, et al. Traumatic Injury to the Superior Mesenteric Artery. American Journal of Surgery 1999; 178 (3): 235-239. 